\title{
Transport of Electronic Excitation Energy in Dye-Loaded Zeolite L
}

\author{
Claudia Minkowski§ and Gion Calzaferri ${ }^{\star}$ \\ $\S$ Mettler Toledo Award Winner (Oral Presentation)
}

\begin{abstract}
Zeolite $L$ is an aluminosilicate with one-dimensional channels, having an opening diameter of $7.1 \AA$. This allows the incorporation of different, electronically non-interacting dye molecules to build an artificial photonic antenna system. The conditions within the channels are such that the dye molecules cannot glide past each other. Hence, a consecutive incorporation leads to crystals with different compartments, where the density of one kind of dye is dominant. The photophysical processes taking place on such dye-loaded zeolite $L$ antenna systems can be studied either on single micrometer or submicrometer sized crystals, on crystals dispersed in a solvent, or coated as thin layers on a support. The energy transfer process that occurs is of the Förster-type and its transfer rate can be tuned, e.g. by first incorporating the acceptor dyes, in a second step consecutively incorporating different amounts of spacer molecules and then in a third step adding the donor dyes. By selectively exciting the donor, energy transfer to the unexcited acceptor as a function of the amount of spacer molecules can be observed. To make a quantitative analysis, one has to take into consideration the situation at the phase boundaries of the particular compartments. This has been done by modeling the distribution of the dye molecules and empty sites within a zeolite crystal by means of a Monte Carlo simulation.
\end{abstract}

Keywords: Energy transfer · Fluorescence $\cdot$ FRET $\cdot$ Host-guest · Supramolecular $\cdot$ Zeolite

\section{Introduction}

Zeolite $\mathrm{L}$ with its one-dimensional channel system is a very versatile material to build artificial host-guest antenna systems. Each of the channels can be filled with different, electronically non-interacting dye molecules that mimic the function of chlorophyll in natural antennae after irradiation with light [1]. A schematic view of the photonic antenna system invented by us is illustrated in Fig. 1. The monomeric dye molecules are represented as rectangles. The dye molecule which has been excited by absorbing an incident photon transfers its electronic excitation energy to a neigh-

${ }^{*}$ Correspondence: Prof. Dr. G. Calzaferri Department of Chemistry and Biochemistry University of Bern

Freiestrasse 3

$\mathrm{CH}-3012$ Bern

Tel.: +41316314236

Fax: +41316313994

E-Mail: gion.calzaferri@iac.unibe.ch boring molecule [2-4]. If the dye molecules interact so that the energy of the excited state is markedly influenced, exciton theory would apply. In the system discussed, however, the interaction is very weak. This kind of energy transport is rather tolerant with respect to defects. After a series of such resonance transport steps, the electronic excitation energy reaches a luminescent trap (dark rectangles). The energy migration is in competition with spontaneous emission, radiationless decay, quenching, and photochemically induced degradation. Fast energy migration is therefore crucial if a trap should be reached before other processes can take place. This means that a detailed understanding of the photochemical and photophysical processes taking place is important. They can be well studied on such dye-loaded zeolite L antenna systems, either on single crystals, on crystals dispersed in a solvent, or coated as thin layers on a support. Another interesting way to investigate the excitation energy transport is the study of $e . g$. dendritic systems [5], or conjugated polymers [6].

We are interested in the energy transport behavior in dye-loaded zeolite L crystals. To know more about the mechanism, we are investigating systems where we assume, experimentally and theoretically, that the zeolite crystals contain donors and accep- tors that are locally separated by different amounts of spacer molecules. This varies the distance $\mathrm{R}$ between donor and acceptor, and we can study the behavior of the excitation energy depending on the amount of spacer molecules (Fig. 2).

For the experiments, we investigate the luminescence properties of such dye-loaded zeolite L crystals. The distribution of dye molecules and empty sites within a zeolite crystal can be studied by means of a Monte Carlo simulation. We report the experimental and theoretical results and discuss their meaning and consistency.

\section{Background}

\section{Host Material}

Zeolite $\mathrm{L}$ is a crystalline aluminosilicate with hexagonal symmetry [7][8]. Its anionic framework and the positions of the chargecompensating cations are illustrated in Fig. $3 \mathrm{a}-\mathrm{d}$. The crystals consist of cancrinite cages ( $\varepsilon$-cages) linked by double six-membered rings. These units form columns in the $c$ direction which are connected, and thus form a twelve-membered ring with a free diameter of $0.71 \mathrm{~nm}$. This gives rise to one-dimensional channels running through the whole crystal, with a largest free diameter of $1.26 \mathrm{~nm}$ and a unit cell length of 0.75 

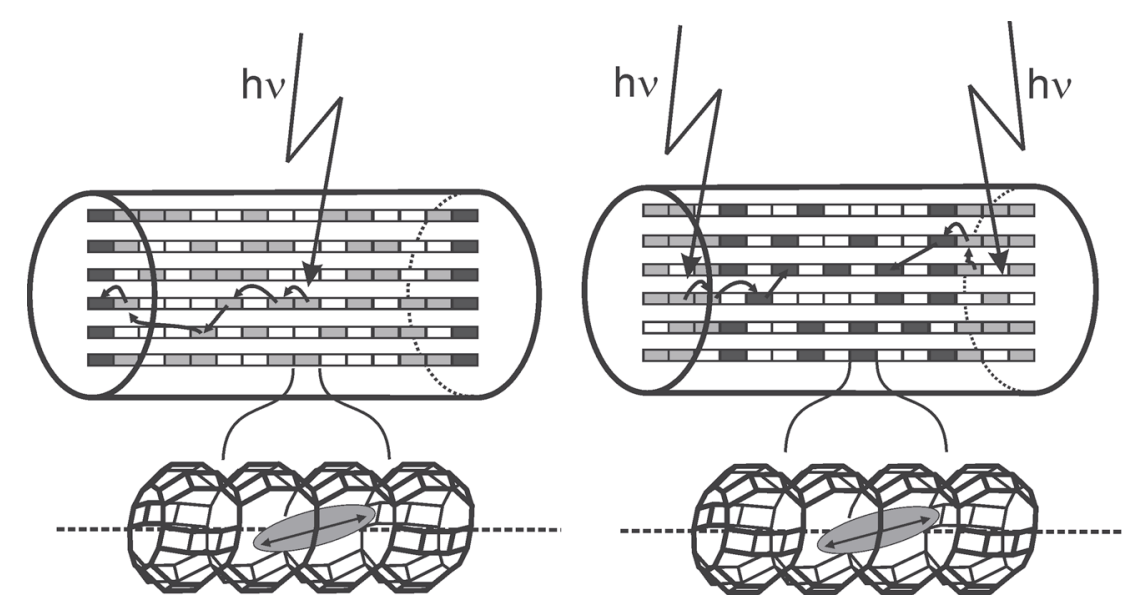

Fig. 1. Representation of a cylindrical nanocrystal consisting of organized dye molecules acting as donors (grey rectangles) and acceptors (black rectangles). The white rectangles represent empty sites. Left: The donors are in the middle part of the crystal and the acceptors at the ends of each channel. Right: The donors are at the ends of each channel and the acceptors are in the middle part. The enlargements show details of the channels with a dye and its electronic transition moments (double-headed arrow) which are parallel to the channel axis for long molecules and tilted for shorter ones.

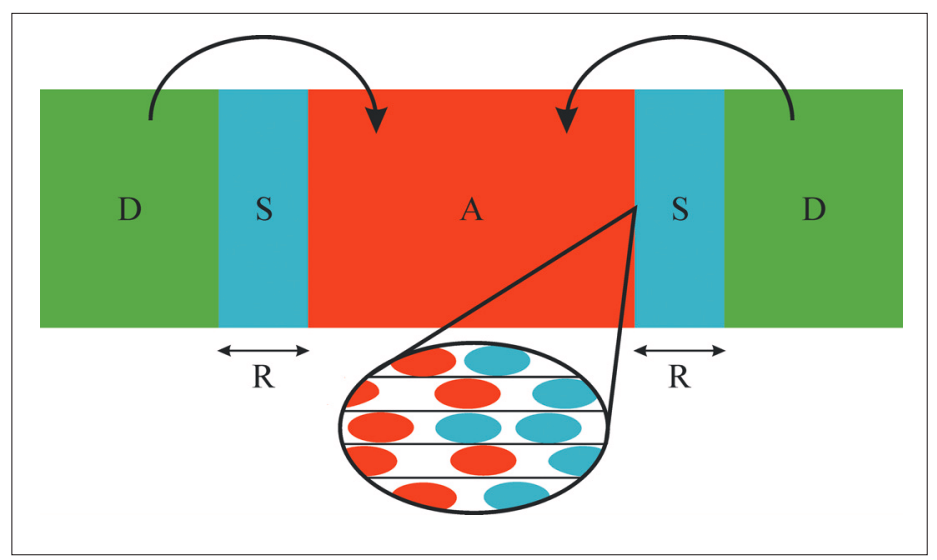

Fig. 2. Scheme of an antenna system with donors $D$ and acceptors $A$ that are separated by different amounts of spacers $S$ to vary the distance $R$ between the donors and the acceptors. The enlargement shows the situation at the interface with the channels of zeolite $L$ indicated by the horizontal lines filled with red (A) or blue (S) ellipses, representing the molecules.

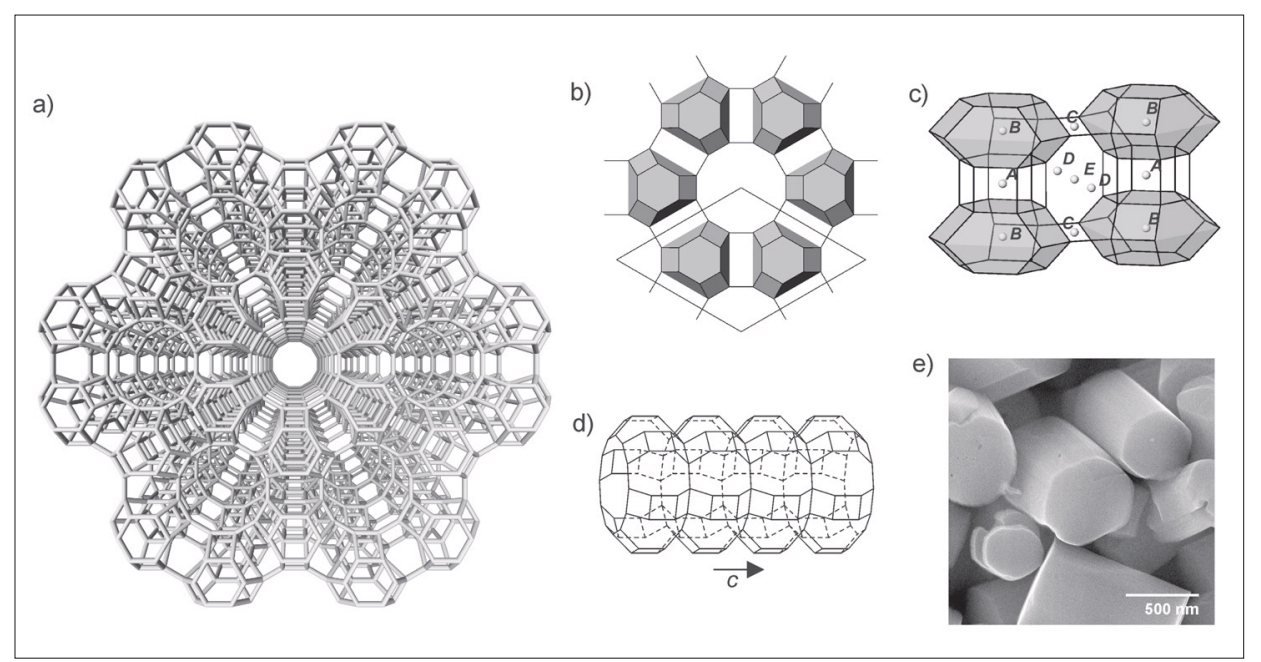

Fig. 3. Zeolite $L$ framework: a) projection along the $c$ axis, b) with the cancrinite cages highlighted as polyhedra, c) section showing the different cationic positions $A$ to $E, d$ ) side view of the 12-ring channel, e) scanning electron microscopy image of zeolite $L$ crystals $\mathrm{nm}$. The main channels are linked via nonplanar eight-membered rings which form an additional two-dimensional channel system with a ring opening of about $0.15 \mathrm{~nm}$. The centre-to-centre distance between two main channels is $1.84 \mathrm{~nm}$. The hexagonal structure of the zeolite $\mathrm{L}$ material is visible in the scanning electron microscopy picture shown in Fig. 3e. This also illustrates that the shape of the crystals can be well described by assuming cylinder morphology [9]. The number of channels lying parallel to the $c$ axis is equal to $0.265\left(d_{c}\right)^{2}$, where $d_{c}$ is the diameter of the cylinder in nanometers. As an example, a crystal with 550 nm diameter consists of about $8 \cdot 10^{4}$ parallel channels. Zeolite L has an anionic framework. Four different cation sites (A, B, C, D) have been reported. $\mathrm{A}$ is in the centre of the double six-membered ring, $\mathrm{B}$ is in the centre of the $\varepsilon$-cage, $\mathrm{C}$ lies midway between the centers of two adjacent $\varepsilon$-cavities, and D is inside the main channel near the wall of the eight-membered ring. Dehydrated zeolite L shows an additional cation site located between two adjacent $\mathrm{A}$ sites and is indicated by $\mathrm{E}$ in Fig. 3c. The water molecules in the large cavities of zeolite L have been reported to behave like an intercrystalline liquid, whereas they seem to build clusters around the cations in the smaller pores [10]. The stoichiometry of zeolite $\mathrm{L}$ with monovalent cations is $(\mathrm{M})_{9}\left[\mathrm{Al}_{9} \mathrm{Si}_{27} \mathrm{O}_{72}\right] \cdot \mathrm{nH}_{2} \mathrm{O}$, where $\mathrm{n}$ equals 21 in fully hydrated materials, and 16 at about $22 \%$ relative humidity.

\section{Dye-loaded Zeolite $L$}

The principle of a dye-loaded zeolite $\mathrm{L}$ antenna system performing radiationless electronic excitation energy migration is shown in Fig. 1. The channels of the zeolite crystal are occupied by energy-transporting dyes (donors) and energy-trapping dyes (acceptors). Strongly luminescent dyes are usually selected. The energy transfer process that occurs when an excited donor $D$ transports its excitation energy to an unexcited acceptor $A$ is of the Förster-type mechanism and its rate constant $k_{E T}$ can be written as in Eqn. (1) [11]

$$
k_{D A}^{F}=\frac{9 \ln (10)}{128 \pi^{5} N_{A} \eta^{4}} \frac{\phi_{D}}{\tau_{D}} J_{D A} G_{D A} p_{D} p_{A}
$$

where $\Phi_{D}$ and $\tau_{D}\left[\mathrm{~s}^{-1}\right]$ are the fluorescence quantum yield and the intrinsic fluorescence lifetime of the donor, $N_{A}$ is Avogadro's number, $\eta$ is the refractive index of the medium, $J_{D A}\left[\mathrm{~cm}^{3} \mathrm{M}^{-1}\right]$ is the spectral overlap integral between the donor emission and the acceptor absorption spectra, and $p_{D}$ and $p_{A}$ are the occupation probabilities of the sites with donors and acceptors. The spectral overlap $J_{D A}$ is equal to the 
integral of the corrected and normalized fluorescence intensity $f_{D}(v)$ of the donor multiplied by the extinction coefficient $\varepsilon_{A}(v)$ of the acceptor (Eqn. (2))

$$
J_{D A}=\int_{0}^{\infty} \varepsilon_{A}(v) f_{D}(v) \frac{d v}{v^{4}}
$$

The term $G_{D A}\left[\AA^{-6}\right]$ expresses the geometrical constraints of the sites in the crystal as a function of the distance $R_{D A}$ between $D$ and $A$, and the relative orientation of the electronic transition moments $K_{D A}$ (Eqn. (3))

$$
G_{D A}=\frac{\kappa_{D A}^{2}}{R_{D A}^{6}}
$$

The distance $R_{D A}$ has a strong effect on the energy-transfer rate constant for the donor-acceptor energy transfer and the donor-donor energy migration. The distance between donors and acceptors can be tuned e.g. by varying the loading of the dye molecules [12] to change the dye-dye distance in general. Another elegant way to study the distance dependence of the excitation energy is to introduce a spacer molecule between the donor and the acceptor molecules to separate them locally. The procedure to prepare such materials has been reported in [13]. To realize such antenna systems, a defined amount of acceptor molecules was first incorporated in the channels of zeolite L. In a second consecutive step, different amounts of spacer molecules were incorporated in the channels of the zeolite crystals already containing the acceptors, and in a third step, the same amount of donor dye was added to the crystals (Fig. 4)). Since the conditions are such that the dyes cannot glide past each other, the crystal is divided into compartments where the density of one dye is dominant. By selectively exciting the donor, energy transfer to the unexcited acceptor as a function of the distance $R_{D A}$ can be observed.

\section{Guest Molecules}

$\mathrm{Py}^{+}$acts as donor and $\mathrm{Ox}^{+}$as acceptor. Due to their favorable spectral properties and their high fluorescence quantum yields, this donor-acceptor pair exhibits remarkable excitation energy transport capability via $\mathrm{Py}^{+}$energy carriers to luminescent $\mathrm{Ox}^{+}$ traps. Their absorption and emission spectra in aqueous solution and in zeolite L differ only little in both environments. The spacer molecules - in our case dye molecules as well - must be chosen so that they do not participate in the energy-transfer process, that is, they should not absorb when the donor is excited and they should not trap the energy from the donor. DMPOPOP was used as spacer molecule, forming the

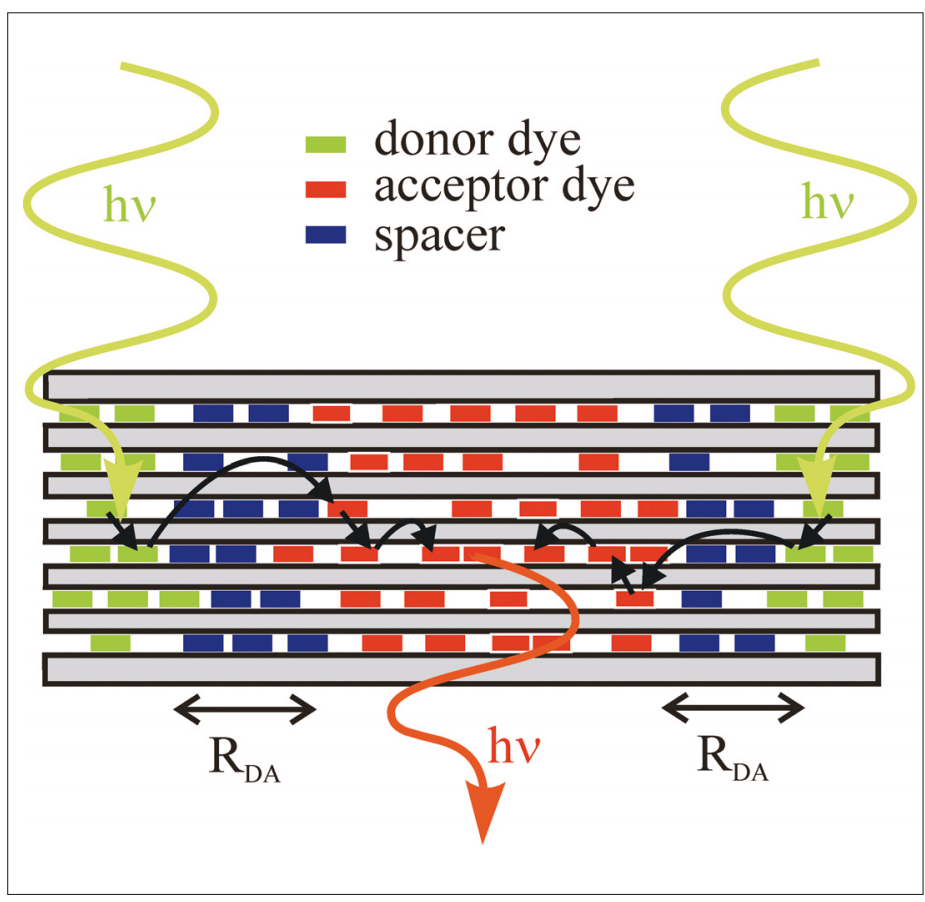

Fig. 4. Schematic picture of a zeolite $L$ crystal with red-emitting dyes in the middle, followed by different amounts of blue spacer molecules forming the so-called spacer layers of different $R_{D A}$, and finally green. emitting donors at the channel endings

$\mathrm{Py}^{+}, \mathrm{DMPOPOP}, \mathrm{Ox}^{+}$-zeolite L antenna system with $\mathrm{Ox}^{+}$in the middle part of the crystal, followed by two DMPOPOP layers and finally $\mathrm{Py}^{+}$at the channel endings. The Table lists the dyes that were used in this work.

The last column of the Table lists the largest interatomic distance of the dye molecules. For $\mathrm{Py}^{+}, \mathrm{Ox}^{+}$, it follows that one dye fits into two unit cells, whereas DMPOPOP occupies three unit cells. Hence, a zeolite channel that is e.g. $600 \mathrm{~nm}$ long contains 400 sites for molecules that fit into two unit cells like $\mathrm{Py}^{+}$and $\mathrm{Ox}^{+}$, and 267 sites for dyes that fit into three unit cells like DMPOPOP. The total number of sites of a zeolite crystal with $600 \mathrm{~nm}$ lengths and $550 \mathrm{~nm}$ in diam- eter is about $3.2 \times 10^{7}$ for $\mathrm{Py}^{+}$and $\mathrm{Ox}^{+}$, and about $2.1 \times 10^{7}$ for DMPOPOP. The occupation probability $p$ of a site containing a dye is equal to the number of occupied sites divided by the number of sites available. This means that $p$ relies on purely geometrical reasoning and that it can be expressed as a function of the dye concentration $c_{d y e}$ per unit volume of a zeolite crystal as expressed in Eqn. (4), where $\rho_{c}$ is the density of the zeolite crystal, $M_{z}$ is the molar mass of a unit cell, and $s$ is the number of unit cells that form one site [14].

$$
p=\frac{M_{z} c_{d y e} S}{\rho_{c}}
$$

Table. Photophysical properties and length of the applied dyes

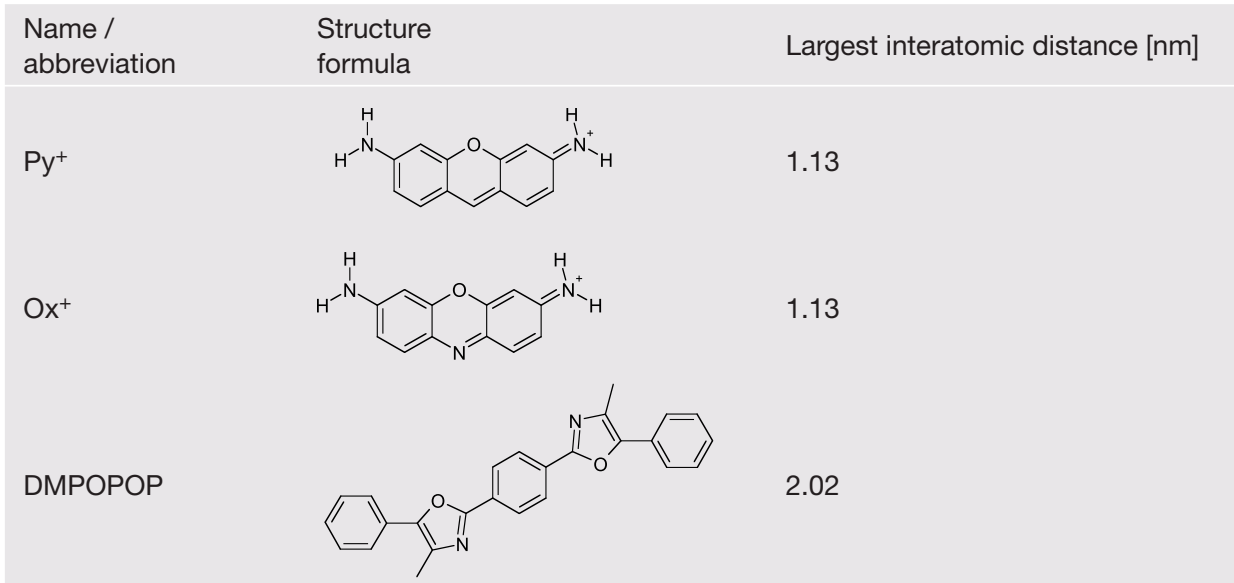



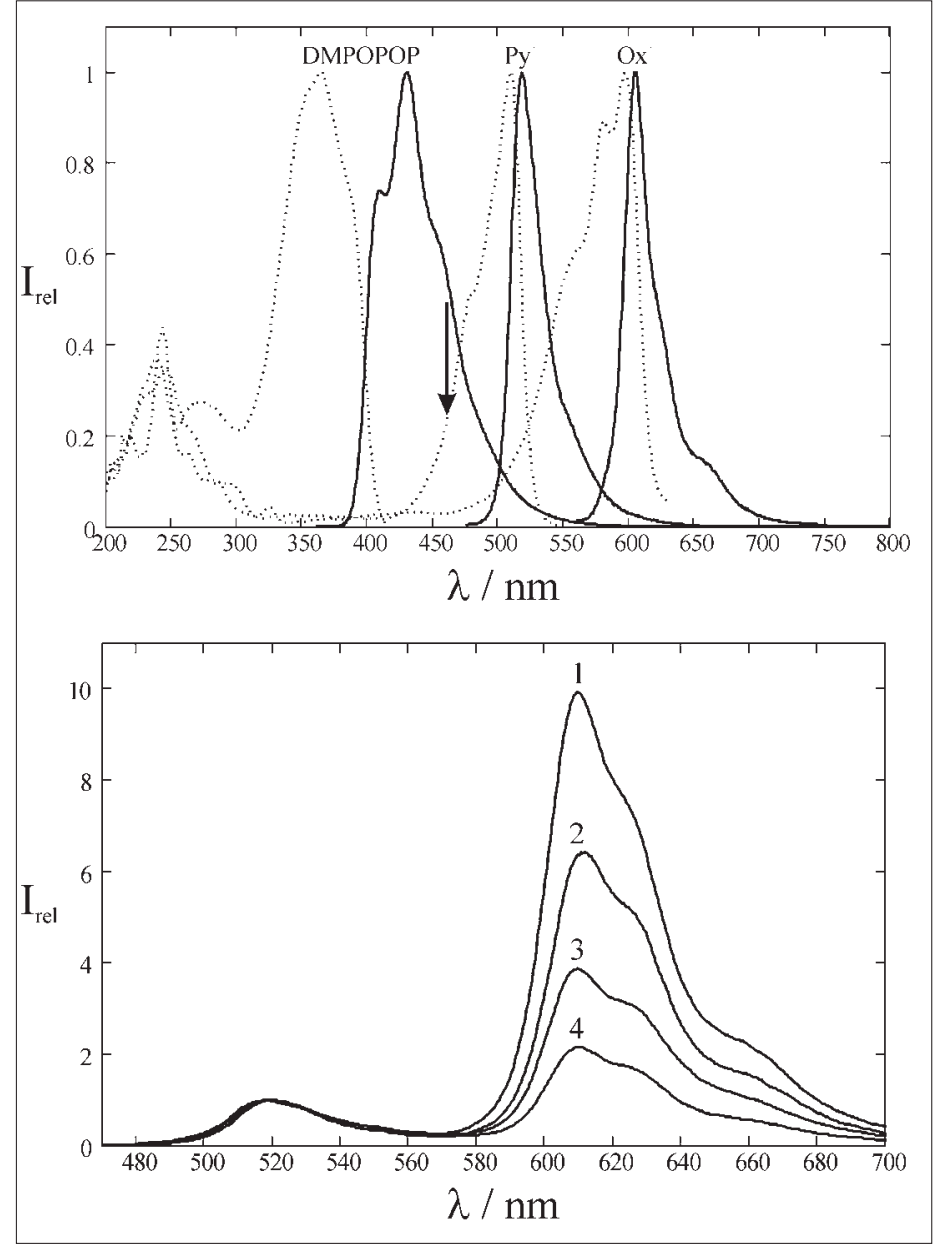

Fig. 5. Top: Excitation (dot) and emission (solid) spectra Py+-zeolite $\mathrm{L}$, DMPOPOP-zeolite L, and $\mathrm{Ox}^{+}$-zeolite $\mathrm{L}$, the arrow indicates the excitation wavelength at $460 \mathrm{~nm}$. Bottom: Fluorescence spectra (scaled to the maximum of the $\mathrm{Py}^{+}$emission) for $\mathrm{Py}^{+}$,DMPOPOP,Ox+ different spacer occupation probabilities $p_{S}$ after selective excitation of $\mathrm{Py}^{+}$ at $460 \mathrm{~nm}$. 1) $\left.\left.\left.p_{S}=0.04,2\right) p_{S}=0.06,3\right) p_{S}=0.085,4\right) p_{S}=0.12$. The inserted amount of $\mathrm{Py}^{+}$and $\mathrm{Ox}^{+}$was kept constant in all samples.
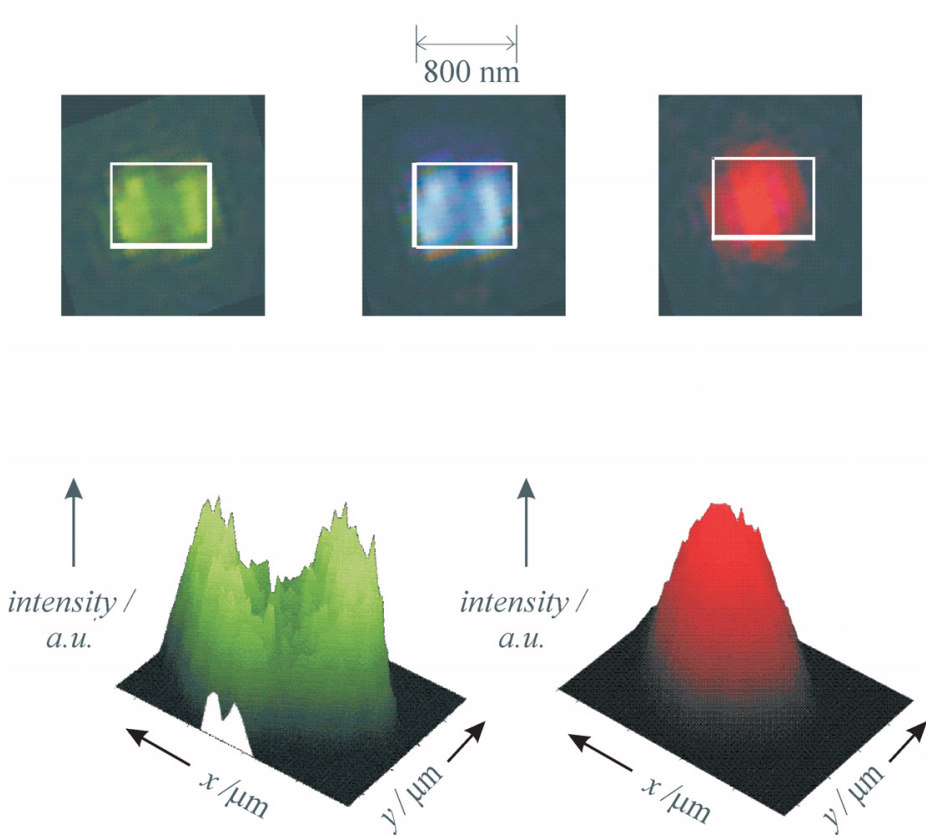

Fig. 6. Upper: fluorescence microscope image of a single zeolite $L$ crystal of $\mathrm{Py}^{+}, \mathrm{DMPOPOP}, \mathrm{Ox}^{+}-$ zeolite $\mathrm{L}$, the edges of which are indicated by the white rectangles. Lower: confocal two-dimensional intensity scan of the $\mathrm{Py}^{+}$and $\mathrm{Ox}^{+}$emission of the same crystal.

\section{Results}

According to the arrangement in Fig. 4, $\mathrm{Py}^{+}$, DMPOPOP,Ox ${ }^{+}$-zeolite L was synthesized. The spacer molecules - in the present case dye molecules as well - must be chosen so that they do not absorb when the donor is excited at $460 \mathrm{~nm}$, and they should not trap the excitation energy. On the other hand, the acceptor should be chosen so that its absorption spectrum overlaps as far as possible with the emission spectrum of the donor. Fig. 5 shows the excitation and emission spectra of the applied dye molecules. For the spectra, each dye was incorporated into zeolite $\mathrm{L}$ in the absence of each other and they were recorded as thin layers on a quartz plate. Appropriate filters were used.

In Fig. 6, we show fluorescence microscopy pictures of an approximately $800 \mathrm{~nm}$ long $\mathrm{Py}^{+}$, DMPOPOP,Ox ${ }^{+}$-zeolite L crystal. Without using a special recording technique that goes beyond the resolution of an optical microscope, we would have obtained a mixture of all colors, due to energy transfer from one dye to the other. But with some carefully developed analytical methods, we can examine the different regions of one single crystal. After specific excitation of the $\mathrm{Py}^{+}$and detection through an interference filter around $550 \mathrm{~nm}$, the green $\mathrm{Py}^{+}$emission can clearly be seen. With a polarizer it is possible, after selective DMPOPOP excitation, to single out the blue DMPOPOP emission which is (unlike the $\mathrm{Py}^{+}$ and $\mathrm{Ox}^{+}$emission) polarized along the crystal axis [15]. Finally, the red $\mathrm{Ox}^{+}$emission can be detected by selectively exciting the $\mathrm{Ox}^{+}$ molecules. The lower images are recorded on a confocal microscope and show two-dimensional plots of the intensity of the $\mathrm{Py}^{+}$and $\mathrm{Ox}^{+}$ emission of the same crystal, after selective excitation.

By simply multiplying the number of spacer molecules with their lengths, the averaged distance $R_{D A}$ can be roughly estimated. The largest interatomic distance of DMPOPOP is $20.2 \AA$, so the values of $R_{D A}$ range from $170 \AA$ to $450 \AA$. The spectral overlap integral and the corresponding Förster radius in parenthesis, calculated for $\kappa_{D A}^{2}=2 / 3$, is ${J_{P y}}^{+}{ }_{-O x}{ }^{+}=$ $2.3 \cdot 10^{-10} \mathrm{~cm}^{6} \mathrm{~mol}^{-1}(77 \AA)$. So the values are far too high. In this system, the donors are dyes that also enter the channels. The dyes cannot glide past each other within one channel, but their positions can overlap with the neighboring channels. This reveals a staggered profile of the phase boundaries. As a consequence, some donor-acceptor pairs lay closer together than anticipated by the values of $R_{D A}$.

\section{Theoretical Reasoning}

\section{Phase Boundaries}

By incorporating different dyes consecutively in the channels of zeolite L, the 
crystal is divided into compartments, where the density of one dye is dominant, since the conditions are such that the dyes cannot glide past each other. But as shown in Fig. 2 , the phase boundaries are relatively diffuse. Therefore we will now focus on the distribution of the dye molecules and on the phase boundaries of the different compartments within the zeolite crystal. We can assume that we have a uniform distribution of the dye molecules in the channels of zeolite L. This proposes a Monte-Carlo calculation, similarly to the approach that was taken for another, analogue system in [14]. The channels of a zeolite L crystal are divided into sites that can be either empty, or occupied by a dye molecule. We take a zeolite L crystal with 1000 channels, each channel containing 300 sites, and fill each of them randomly with 90 dye molecules on average. The resulting occupation probability $p$ for this dye-loaded zeolite L compound is then 0.3 . If we plot the occupation probability $p$ of these 1000 channels having an average loading of 0.3 and normalize the incidences to 1 (y-axis) (Fig. 7) it shows that a Gaussian distribution results in a mean loading of $p=0.3$.

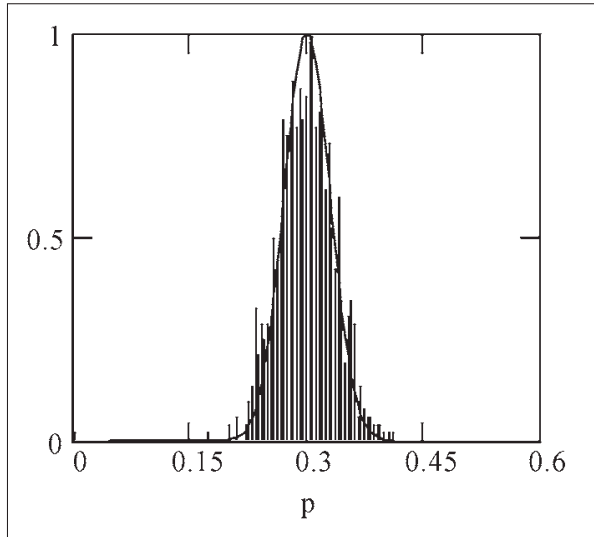

Fig. 7. Diagram of the Gaussian distribution of dye-loaded zeolite channels. The bars represent the amount of channels having a certain occupation probability $p$, normalized to one. The Gauss function is plotted as a solid line.

We now look at a crystal with 40 parallel channels, each channel having 60 sites. We then randomly distribute one kind of dye molecules among these sites with an average occupation probability $p=0.3$. A schematic image of such a zeolite crystal is shown in Fig. 8 (top). The dye molecules are represented as dark dots. In a consecutive step, we add another type of dye molecule. Therefore, we virtually seal the channels at the right side of the crystal and fill the channels only from the left side. This is a simplification we can apply without loss of information. In the experiment we have the first dyes that are pushed further into the centre of the crystal, and the second dyes enter at both crystal endings. But

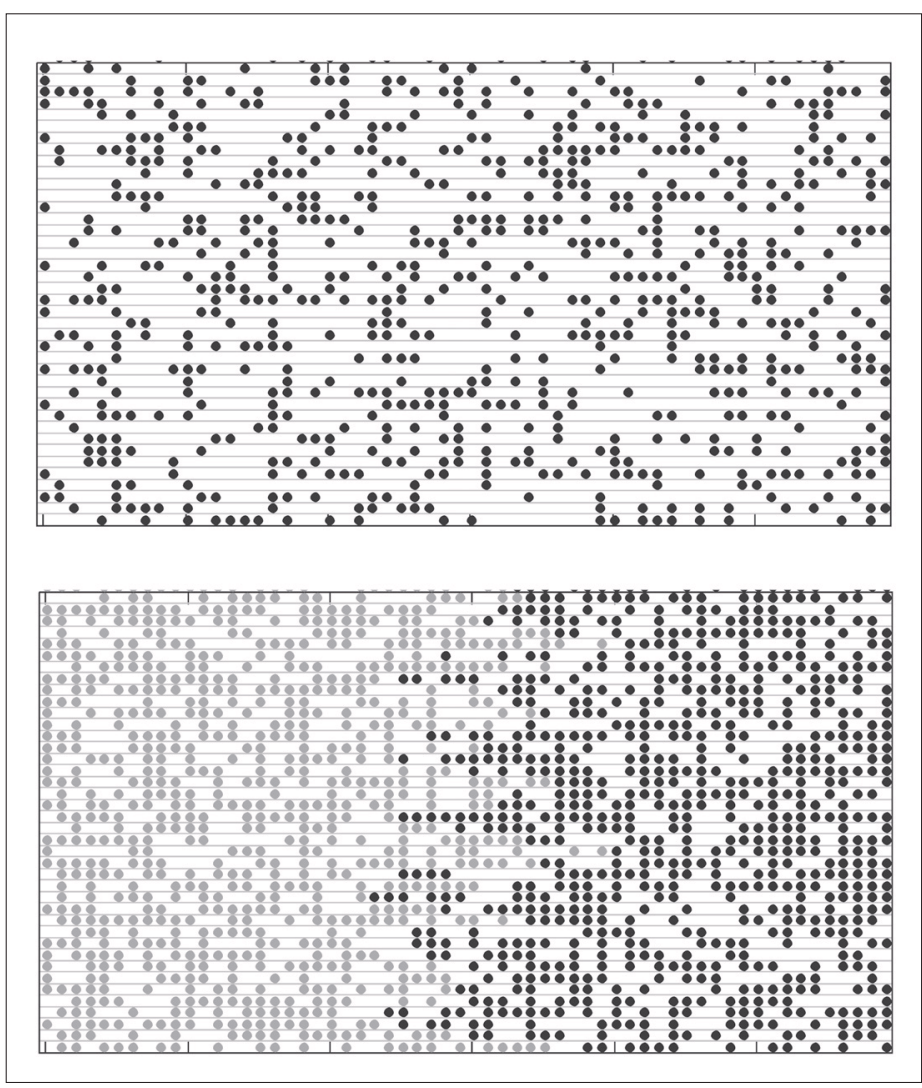

Fig. 8. Simulated images of two zeolite L crystals, each containing 40 parallel channels, every channel containing 60 sites. Top: The sites are randomly filled with dye molecules (dark dots). The occupation probability $p$ of the dye molecules equals 0.3 . Bottom: The sites are first randomly filled with dye molecules (dark dots), while after virtually sealing the right channel entrances, another type of dye (light dots) was consecutively filled in the channels from the left side. The occupation probability $p$ for both dyes equals 0.3 , so the overall occupation probability of this zeolite crystal is 0.6 . The phase boundaries extend over 10 to 12 sites.

we now fill the same amount of a second type of dye molecules from the left side. This case is shown Fig. 8 (bottom), where the second type of dye molecules is represented as light dots. We notice the increase of the dye content. The overall occupation probability increases to 0.6 . A close look at the phase boundary reveals the staggered profile of the borders. The swapping area for this dye concentration extends over ten to twelve sites. If we consider one site to include two unit cells, this spans an area of 12 to $18 \mathrm{~nm}$.

\section{Discussion}

We found that the spacer-loading $p_{S}$ and therefore the distance $R_{D A}$ has a strong effect on the energy transfer rate, as can be seen in the fluorescence intensities of the acceptor in samples where $p_{S}$ is different. The estimated values of $R_{D A}$ are in a range that is far too high due to the staggered profile of the phase boundaries. As a consequence, some donor-acceptor pairs lay closer together than anticipated by the values of $R_{D A}$. The phase boundaries can be studied by modeling the distribution of dye molecules and empty sites within a zeolite crystal with a Monte-Carlo simulation. This also shows that the swapping area between two phase boundaries is relatively large. Replacing the donors by so-called stopcock molecules that have a tail that enters the channel and a head that is to large to enter results in a much less diffuse phase boundary. Then, all stopcock donors are fixed at the same position and only one staggered phase boundary from the spacer to the acceptor remains [16].

\section{Experimental Section}

\section{Materials}

Zeolite L crystals were synthesized and characterized as described in [17]. The potassium-exchanged form was used, with an average crystal length and diameter of $840 \mathrm{~nm}$ and $750 \mathrm{~nm}$, respectively. $\mathrm{Py}^{+}$and $\mathrm{Ox}^{+}$were synthesized and purified. DMPOPOP from Fluka was used without further purification. 
$P y^{+}, D M P O P O P, O x^{+}$-zeolite $L: \mathrm{Ox}^{+}$was first incorporated into the channels of zeolite $\mathrm{L}$ via ion exchange in water. Therefore, zeolite L was suspended in water and sonicated for $20 \mathrm{~min}$ to avoid aggregation of the crystals. Then, an aq. soln. of $\mathrm{Ox}^{+}$containing the required amount of $\mathrm{Ox}^{+}$was added and refluxed $7 \mathrm{~h}$ at $100^{\circ} \mathrm{C}$. The $\mathrm{Ox}^{+}$-loaded zeolite L crystals were washed with methanol to remove the $\mathrm{Ox}^{+}$molecules quantitatively from the crystal surface. The neutral DMPOPOP was then inserted from the gas phase. The $\mathrm{Ox}^{+}$-loaded zeolite L crystals were transferred in a glass ampoule and dehydrated under vacuum $\left(3 \cdot 10^{-2}\right.$ mbar $)$ at $80{ }^{\circ} \mathrm{C}$ for $24 \mathrm{~h}$ to empty the adsorption sites in the channels. Then, the required amount of DMPOPOP was added and the ampoule was sealed. The incorporation took place by heating the ampoule in a rotating furnace at $150{ }^{\circ} \mathrm{C}$ for $6 \mathrm{~d}$. Afterwards, the DMPOPOP, $\mathrm{Ox}^{+}$-zeolite L crystals were washed with $n$-butanol and rehydrated in an exsiccator containing a saturated solution of potassium acetate. Finally, $\mathrm{Py}^{+}$was again incorporated from water, according to the procedure for $\mathrm{Ox}^{+}$, but refluxed for only $30 \mathrm{~min}$.

\section{Physical Characterization \\ Thin Layer Preparation}

$100 \mu \mathrm{L}$ of dye-loaded zeolite L suspended in n-butanol $(\sim 1 \mathrm{mg}$ in $300 \mu \mathrm{l})$ was dropped on a quartz plate $(\varnothing 1.6 \mathrm{~cm})$ and the solvent was evaporated in air. The quartz plate was then covered with a Petri dish.

\section{Steady-state Absorption and Fluores- cence Measurements \\ Emission and excitation spectra were re- corded on a Perkin Elmer LS 50B lumines- cence photospectrometer under application of appropriate cut-off filters. The samples were measured as thin layers.}

\section{Optical Fluorescence Microscopy}

The fluorescence microscopy images were recorded on an Olympus BX 60 device equipped with a Kappa CF 20 DCX Air K2 CCD camera. Light of a Xe-lamp was focused on the sample. To generate the green $\mathrm{Py}^{+}$ emission, the crystal was excited at 470-490 $\mathrm{nm}$ and the emission was detected through a broad-band interference filter at $550 \mathrm{~nm}$. The blue DMPOPOP emission was recorded by exciting the DMPOPOP at 330-385 nm and only transmitting emission polarized in the direction of the crystal axis. The red $\mathrm{Ox}^{+}$ emission was detected after excitation at $545-580 \mathrm{~nm}$. The confocal microscopy pictures were recorded on an Olympus fluoview 300 confocal laser scanning microscopy system, equipped with an Ar- and a He:Ne-laser for the excitation [18].

\section{Acknowledgements}

This work was supported by the Schweizerischer Nationalfonds zur Förderung der wissenschaftlichen Forschung NF 200020105140/1 and the Swiss National Science Foundation NFP 47 (4047-057481).

Received: January 4, 2005

[1] T. Ritz, A. Damjanović, K. Schulten, ChemPhysChem 2002, 3, 243.

[2] T. Förster, 'Fluoreszenz Organischer Verbindungen', Vandenboeck \& Ruprecht Göttingen, 1951.

[3] D.L. Andrews, 'Resonance Energy Transfer', John Wiley \& Sons, New York, 1999.

[4] J.R. Lakovicz, 'Principles of Fluorescence Spectroscopy', 2nd ed., Kluwer Academic-Plenum Publishers NY, 1999.

[5] D.J. Heijs, V.A. Malyshev, J. Knoester, $J$. Chem. Phys. 2004, 121, 4884.

[6] D. Beljonne, G. Pourtois, C. Silva, E. Hennebicq, L.M. Herz, R.H. Friend, G.D. Scholes, S. Setayesh, K. Müllen, J.L. Brédas, PNAS 2002, 99, 10982.
[7] C. Baerlocher, W.M. Meier, D.H. Olson, 'Atlas of Zeolite Framework Types', 5th ed., Elsevier, Amsterdam, 2001.

[8] T. Ohsuna, B. Slater, F. Gao, J. Yu, Y. Sakamoto, G. Zhu, O. Terasaki, D.E.W. Vaughan, S. Qiu, C.R.A. Catlow, Chem. Eur. J. 2004, 10, 5031.

[9] O. Larlus, V.P. Valtchev, Chem. Mater. 2004, 16, 3381.

[10] D.W. Breck, 'Zeolite Molecular Sieves', John Wiley \& Sons, New York, 1974.

[11] N. Gfeller, G. Calzaferri, J. Phys. Chem. B 1997, 101, 1396.

[12] S. Megelski, G. Calzaferri, Adv. Funct. Mater. 2001, 11, 277.

[13] M. Pauchard, A. Devaux, G. Calzaferri, Chem. Eur. J. 2000, 6, 3456.

[14] M.M. Yatskou, M. Meyer, S. Huber, M. Pfenniger, G. Calzaferri, ChemPhysChem 2003, 4, 567.

[15] S. Megelski, A. Lieb, M. Pauchard, A. Drechsler, S. Glaus, C. Debus, A.J. Meixner, G. Calzaferri, J. Phys. Chem. B 2001, $105,25$.

[16] C. Minkowski, G. Calzaferri, submitted for publication.

[17] A. Zabala, D. Brühwiler, T. Brun, G. Calzaferri, Monatshefte für Chemie 2005, 136, 77

[18] G. Calzaferri, S. Huber, H. Maas, C. Minkowski, Angew. Chem. Int. Ed. 2003, 42, 3732 . 